\title{
Demonstration of agglutinating anti-Leishmania antibodies in lymph node aspirate for confirmation of kala-azar serodiagnosis
}

Despite poor sensitivity for the diagnosis of visceral leishmaniasis (VL),

demonstration of amastigotes in Giemsa-stained organ aspirate is still considered the 'gold standard' (WHO, 2004). As it is less risky and easily applicable in rural health settings compared with bone-marrow or spleen aspiration, lymph node aspiration is routinely carried out in many VL endemic areas. However, even with the incorporation of highly sensitive techniques such as PCR, the reliability of lymph-node aspiration for VL diagnosis, although improved in advanced disease, nevertheless remains below the desired level in early and mild infections (Osman et al., 1997).

Based on the knowledge that lymphadenopathy and adenomegaly are among the most important clinical features in VL infection, efforts to diagnose the disease have been exclusively concentrated on the demonstration of amastigotes in lymph aspirates (Babiker et al., 2007).

However, since lymph nodes constitute the first immunological barriers against Leishmania invasion as well as being the principal sites where immune reactions are initiated, it might also be of interest to evaluate their contribution to the coordinated immune defence developing within the host.

Our aim here is to determine whether the expected antibody response in lymph aspirates corroborates results obtained with serum for diagnosis of VL, particularly in patients presenting typical disease symptoms but with negative microscopy for Leishmania donovani.

The study was carried out in Doka peripheral hospital, a well-known VL endemic focus in Gedarif State (Eastern Sudan), with the permission of the Kala-azar National Control Programme Coordinator (Sudanese Ministry of Health). In total, $48 \mathrm{VL}$ suspects were received during the period February-April
2006. Besides fever (duration $\geqslant 2$ weeks), splenomegaly and lymphadenopathy were among the most prevalent manifestations. Patients and/or their guardians were informed about the possibility of VL infection and the procedures to be followed for diagnosis.

For preparation of sera, blood samples were collected from all patients. Lymph aspiration was performed without administration of anaesthetic. After cleaning the site of the inguinal lymph node with alcohol, an aspiration puncture was made with a commercial Vacutainer needle holder and 21-gauge needle as for blood collection. Keeping the needle in the node and applying constant suction in different directions, aspirates $(20-30 \mu \mathrm{l})$ were collected from which minute portions (3-5 $\mu \mathrm{l}$ ) were dispensed in cryo-tubes; the remaining portions of the specimens were smeared on to microscope slides.

Following air drying and fixation with methanol, the smears were stained with Giemsa. Lymph specimens designated for the direct agglutination test (DAT) were transported in ice to the central laboratory (Omdurman) where they were stored at $-20{ }^{\circ} \mathrm{C}$ until required.

Preparation of the standard $[\beta$ mercaptoethanol ( $\beta$-ME)-treated formaldehyde-fixed] DAT antigen (FF) was as described in previous reports (el Harith et al., 1995, 2003). For comparison of reactivity against the FF antigen in lymph specimens, a commercial trypsin-treated freeze-dried (FD) version (Meredith et al., 1995) was included. The DAT on the serum samples collected from all 48 patients with suspected VL (peripheral hospital, Doka) was according to the improved procedure using $\beta$-ME to eliminate non-specific agglutination (el Harith et al., 1995). Besides testing by the improved DAT procedure as for serum (central laboratory, Omdurman), duplicates of lymph specimens collected from the 48 patients with suspected VL were also tested by the original method without $\beta$-ME treatment (el Harith et al., 1986). For confirming results with the FF antigen, lymph specimens from 16 patients with suspected VL were tested against the FD antigen following instructions provided by the manufacturer for serum testing (Meredith et al., 1995). Test results with the FF or FD antigen were read $18 \mathrm{~h}$ later, taking a 1:3200 titre as the VL cut-off for both serum and lymph specimens.

Final VL diagnosis was either by demonstration of amastigotes in stained lymph smears or by positive serum DAT result (titres $\geqslant 1: 3200$ ) combined with positive presentation of the disease (WHO, 2004). The patients diagnosed with VL were put on a 30-day treatment course of sodium stibogluconate (Pentostam; Albert David). Patients with disorders other than VL were either treated accordingly in the peripheral health setting or referred to the state hospital in Gedarif for further investigations.

The results of microscopy and of the DAT with serum or lymph aspirates obtained from the 48 patients with suspected VL are summarized in Table 1. All 17 VL cases identified by either microscopy (10) or positive DAT results in serum (7) responded favourably to Pentostam therapy. Of the 31 patients excluded from VL infection, 3 were successfully treated with antimalarials and 4 with antibiotics against pneumonia (2) or typhoid (2); the remaining 24 patients were referred to the district hospital in Gedarif.

Except for a fourfold increase in titre in the lymph specimen from one VL patient, no significant improvement due to $\beta$-ME incorporation was observed in the titre levels of the remaining 16 (results not shown). Despite reduction with $\beta$-ME, titres in the non-VL lymph specimens (31) remained unchanged below the VL cut-off. Titres obtained in 7 VL lymph specimens tested with the (trypsinized) FD antigen 
Table 1. Agglutinating antibody titres measured in lymph node aspirates and sera from 48 patients with suspected VL

\begin{tabular}{|lcll|}
\hline $\begin{array}{l}\text { No. of } \\
\text { patients } \\
\text { examined }\end{array}$ & $\begin{array}{c}\text { Parasitological } \\
\text { diagnosis (lymph node } \\
\text { aspirate smear) }\end{array}$ & \multicolumn{2}{c|}{ DAT titre range } \\
\cline { 3 - 4 } & & Lymph node aspirate & \multicolumn{1}{c|}{ Serum $^{\star}$} \\
\hline 4 & Positive & $\geqslant 1: 102400$ & $\geqslant 1: 102400$ \\
6 & Positive & $1: 12800-1: 51200$ & $\geqslant 1: 102400$ \\
5 & Negative & $1: 3200-\geqslant 1: 102400$ & $1: 25600-\geqslant 1: 102400$ \\
2 & Negative & $1: 800-1: 1600$ & $1: 12800-1: 51200$ \\
2 & Negative & $1: 1600$ & $1: 100,1: 1600$ \\
7 & Negative & $\leqslant 1: 200$ & $\leqslant 1: 400$ \\
22 & Negative & $\leqslant 1: 200$ & $\leqslant 1: 200$ \\
\hline
\end{tabular}

${ }^{\star}$ Specimens tested after reduction with $\beta$-ME.

were almost identical to those with the standard ( $\beta$-ME-treated) FF antigen (results not shown). Both antigens scored negative DAT readings in the 9 lymph specimens from non-VL patients.

This is the first VL study in which lymph specimens primarily collected for amastigote demonstration were simultaneously assessed for anti-Leishmania antibody response. Whether the response is systemic, involving all lymphoid organs, or local, triggered by amastigotes trapped in the individual nodes, is beyond the scope of this study. By comparison with blood (serum), which receives a direct and generous supply of anti-Leishmania antibodies from the spleen, lymph drained from individual nodes is expected to contain relatively lower concentrations. Nonetheless, all 10 lymph specimens from the confirmed VL cases scored DAT titres above the cut-off, implying suitability for VL detection comparable to serum or Giemsa-stained lymph smears (Table 1).

Of particular interest, however, were the diagnostic titres $(\geqslant 1: 3200)$ measured in serum as well as in lymph specimens from five unconfirmed VL cases, indicating superiority over tissue smears for VL detection possibly at an early stage. Probably due to the sampling of a single (inguinal) node, employing a VL cut-off identical to that previously established for serum (titre $=1: 3200$ ) and the possibility of early or subclinical infection, two of the (sero-positive) unconfirmed VL cases reacted at a negative or marginal titre
( $1: 800$ or $1: 1600)$. Concordant nevertheless with serum was the specificity of the antibodies detected in all lymph specimens from the VL patients as evidenced by the stability in titre levels despite reduction with $\beta$-ME. The relevance of measuring antibody response in lymph to support VL differential diagnosis is further substantiated by the unambiguous negative DAT readings obtained in 29 of the 31 patients that had a clinical presentation highly indicative of VL.

Despite the limited number, lymph specimens tested against the trypsinized antigen showed DAT readings almost identical to those of the $\beta$-ME treated, corroborating the eligibility of lymph for the demonstration of anti-Leishmania antibodies.

Similar evaluation in bone-marrow aspirates and employing detection methods other than DAT is expected to reveal additional diagnostic information. However, unlike bone-marrow or spleen aspiration, lymph node aspiration requires no special precautionary measures and can easily be performed in rural health settings. Specimen volumes of 20-30 $\mu \mathrm{l}$ sufficient for preparation of tissue smears and for DAT can be obtained from a single node. As for blood, lymph aspirates can also be spotted on to filter papers and after air drying stored at $+4{ }^{\circ} \mathrm{C}$ or $-20{ }^{\circ} \mathrm{C}$ until required.

Future plans for improving sensitivity include sampling of both inguinal nodes, and incorporation of appropriate lysis buffer to ensure optimal homogenization of lymph specimen and denaturant (urea) to effectively eliminate non-specific agglutination so that a lower VL cut-off titre $(1: 200-1: 400)$ can be adopted (el Harith et al., 1995; el Mutasim et al., 2006).

\section{Acknowledgements}

We wish to thank Dr Gasim Badri (President), Dr Farouk Abdulaziz (Dean of School of Medicine) and Dr Abubaker Uro (Head of the Ahfad Centre for Science and Technology), Ahfad University for Women, for facilitating execution of this study. Our thanks also go to Professor M. Mukhtar and Dr W. el Amin, Institute of Endemic Diseases, University of Khartoum, for their help in the field work, cryo-preservation and maintenance of the $L$. donovani strain.

\section{Elfadil M. Abass, ${ }^{1}$ Durria Mansour ${ }^{1}$ and Abdallah el Harith ${ }^{2}$}

${ }^{1}$ Ahfad University for Women, PO Box 167, Omdurman, Sudan

${ }^{2}$ Wijngaard 155, 8212 CJ Lelystad, The Netherlands

Correspondence: Abdallah el Harith (harith17@yahoo.com)

Babiker, Z. O. E., Davidson, R., Mazinda, C., Kipngetch, S. \& Ritmeijer, K. (2007). Utility of lymph node aspiration in the diagnosis of visceral leishmaniasis in Sudan. Am J Trop Med Hyg 76, 689-693.

el Harith, A., Kolk, A. H. J., Kager, P. A., Leeuwenburg, J., Mnigai, I., Kiugu, S. \& Laarman, J. J. (1986). A simple and economical direct agglutination test for serodiagnosis and sero-epidemiological studies of visceral leishmaniasis. Trans $\mathrm{R}$ Soc Trop Med Hyg 80, 583-587.

el Harith, A., Chowdhury, C., Al-Masum, M., Samiao-Santos, S. J., Das, P. K., Akhter, S., Vetter, J. C. M. \& Haq, I. (1995). Evaluation of cleaving agents other than trypsin in direct agglutination test for further improving diagnosis of visceral leishmaniasis. J Clin Microbiol 33, 1984-1988.

el Harith, A., el Mutasim, M., Mansour, D., Mustafa, E. F. \& Arvison, H. (2003). Use of glycerol-preserved antigen as an alternative to freeze-drying for long-term preservation of antigen for the direct agglutination test. Trop Med Int Health 8, 1025-1029.

el Mutasim, M., Mansour, D., Abass, E. M., Hassan, W. M. \& el Harith, A. (2006). Evaluation of a glycerol-preserved antigen in the direct agglutination test for the diagnosis of visceral 
leishmaniasis at rural level in eastern Sudan. $J$ Med Microbiol 55, 1343-1347.

Meredith, S. E., Kroon, N. C., Sondorp, E., Seaman, J., Goris, M. G. A., van Ingen, C. W., Oosting, H., Schoone, G. J., Terpstra, W. J. \& Oskam, L. (1995). Leish-KIT, a stable direct agglutination test based on freeze-dried antigen for serodiagnosis of visceral leishmaniasis. J Clin Microbiol 33, 1742-1745.

Osman, O. F., Oskam, L., Zijlstra, E. E., Kroon, N. C., Schoone, G. J., Khalil, E. T., El-Hassan, A. M. \& Kager, P. A. (1997). Evaluation of PCR for diagnosis of visceral leishmaniasis. J Clin Microbiol 35, 2454-2457.

WHO (2004). Report of the Scientific Working Group Meeting on Leishmaniasis, Geneva, 2-4 February 2004. TDR/SWG/04. Geneva: World Health Organization. 